\title{
Fixed violaceous fingertips in a young girl with anxious hair twirling habit
}

\author{
Jeffrey Morris*, Leeor Porges and Ana Duarte \\ NSU COM Research Fellowship Program, Nova Southeastern University, USA
}

\section{Introduction}

Hair tourniquet was described over 300 years ago but it is still commonly overlooked by physicians today. It most commonly occurs before puberty when a hair or thread strangulates a distal appendage, compromising venous and lymphatic drainage. If the constriction persists long enough, arterial blood supply can be compromised resulting in ischemia and possible gangrene. In this case report we describe a tourniquet-like syndrome but it differs from the other cases described in the literature due to the patient inflicting it upon herself, resulting in an intermittent strangulation of multiple digits. The mechanism likely differs because instead of the increase pressure causing a cyanosis turning the digits blue, we suspect the increased pressure brought about by the tourniquet is forcing red blood cells into the interstitium turning the tips of the digits dark purple.

\section{Case report}

A four year old girl with a congenital solitary kidney presented to the dermatologist with permanent dark purple pigmentary changes on her fingertips (Figure 1). There was no evidence of acute trauma, but her parents reported that she had a nervous habit of twirling any string, rubber band or hair around her fingers causing constriction. Changes in color were found on the tips of digits one through four bilaterally; distal phalanges felt warm to the touch. The violaceous areas were fixed and unaltered by temperature or scrubbing preparations; no changes in color were noted with warming. The remainder of the physical exam was unremarkable. The patient had no known cognitive deficits. The patient had no other visible skin lesions and no palpable nodules. On further examination there was no livedo reticularis and no signs of perniosis. On the dorsal side of the hand no dropouts of periungual vasculature were noted. When examined under dermoscopy, there

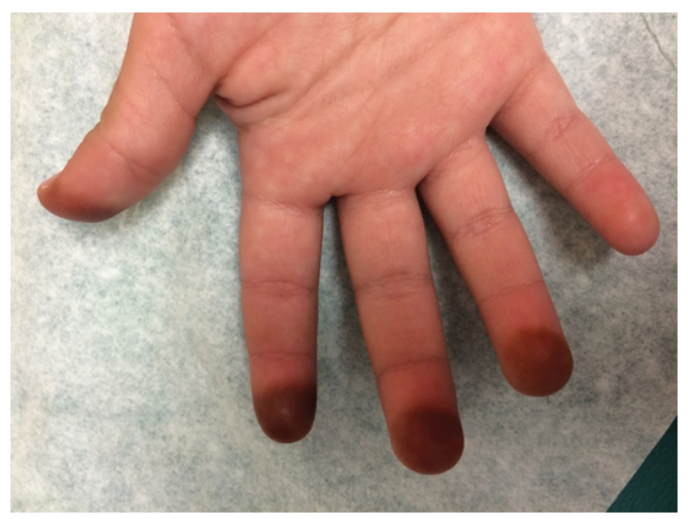

Figure 1. Fixed violaceous fingertips. were no dilation of vessels; however, pigment was present on the ridges surrounding the acosyringium on the palmer side of the digit pads.

\section{Discussion}

This is the first reported case of self-inflicted hair-thread-tourniquet syndrome in a four-year-old girl. Non-accidental causes of cyanosis due to tourniquet syndrome in youth are typically attributed to child abuse [1]. Involvement of more than one digit is typically associated with intentional causes [2]. In this case, the attending physician witnessed the child's nervous habit, and noted a purple discoloration at the tips of the first few digits bilaterally. Edema and a bluish discoloration at the tip of the digit has been described in other case reports where a hair becomes wrapped around a digit [3], but a different mechanism due to the intermittent pathophysiology could account for the lack of edema in this case. The process of increased interstitial pressure causing digits to change color usually occur over hours to weeks, but duration between the time of injury and time of first detection has been reported to be as long as 3 months [4]. When a discontinuous mechanism exists, this process can take much longer, making it difficult to determine how long this constriction has been occurring. No biopsy was taken; we felt it could do more harm than good to the child.

The diagnosis was made clinically but we recommend ruling out other possible acute causes of blue fingers. Primary Raynaud disease is intermittent cyanosis due to vasospasm. Characteristics that physicians look for to help diagnose primary Raynaud is a young age of onset, symmetrical bilateral blue fingers, and the absence of a detectable underlying cause [5]. Perhaps the intermittent nature of the former disease makes it less likely than acrocyanosis, which is defined as a permanent blue discoloration of the extremities. While there is still much we do not know about acrocyanosis pathophysiologicaly, the existing hypothesis also suggests that a vasospastic reaction is taking place [6]. Vasospasm is unlikely in this patient given that his fingers were warm to the touch, making Raynauds phenomenon and acrocyanosis unlikely. The fact that this patient exhibits renal pathology could put cryoglobulinemia on our list of differentials. One French study of 36 patients with type I cryoglobulinemia found that $75 \%$ of patients showed skin or vasomotor symptoms such as blue digits and $30 \%$ had nephropathy, demonstrating a possible association [7]. However, cryoglobulins are immunoglobulins that reversibly precipitate at low temperature, so we would expect the proteins to de-precipitate once re-

Correspondence to: Jeffrey Morris, Research Fellow, NSU COM Research Fellowship Program, USA, E-mail: Jm3463@nova.edu

Received: February 25, 2016; Accepted: March 06, 2016; Published: March 08, 2016 
exposed to warm temperatures. Lupus and certain vasculitis also have renal and vasomotor symptoms.

Arriving at the correct diagnosis is imperative so that the most beneficial treatment can be started. It is not often a dermatologist must make a referral to a mental health professional, but given the nervous symptoms exhibited by the patient, consultation with a psychiatrist or psychologist for behavioral treatment is first line therapy. Medical treatment available later includes selective serotonin reuptake inhibitors which may be helpful. It is important to first rule out acrocyanosis because medications that can increase the blood concentrations of serotonin have been reported to cause or worsen acrocyanosis in the pediatric population [8]. Other medications that may help restore normal blood flow include vasodilators such as antihypertensives, phosphodiesterase type 5 inhibitors and nitroglycerin.

\section{References}

1. Klusmann A, Lenard HG (2004) Tourniquet syndrome--accident or abuse? Eur $J$ Pediatr 163: 495-498. [Crossref]
2. Sivathasan N, Vijayarajan L (2012) Hair-thread tourniquet syndrome: a case report and literature review. Case Rep Med 2012: 171368. [Crossref]

3. Sudhan ST, Gupta S, Plutarco C (2000) Toe-tourniquet syndrome--accidental or intentional? Eur J Pediatr 159: 866. [Crossref]

4. Sunil TM (2001) The Hair-Thread-Tourniquet Syndrome - report of an unusual presentation of this rare condition. Hand Surg 6: 231-233. [Crossref]

5. Bakst R, Merola JF, Franks AG Jr, Sanchez M (2008) Raynaud's phenomenon: pathogenesis and management. J Am Acad Dermatol 59: 633-653. [Crossref]

6. Das S, Maiti A (2013) Acrocyanosis: an overview. Indian J Dermatol 58: 417-420 [Crossref]

7. Néel A, Perrin F, Decaux O, Dejoie T, Tessoulin B, et al. (2014) Long-term outcome of monoclonal (type 1) cryoglobulinemia. Am J Hematol 89: 156-161. [Crossref]

8. Karakaya I, Aydoğan M, Coşkun A, Gökalp AS (2003) Acrocyanosis as a side effect of tricyclic antidepressants: a case report. Turk J Pediatr 45: 155-157. [Crossref]

Copyright: (C2016 Morris J. This is an open-access article distributed under the terms of the Creative Commons Attribution License, which permits unrestricted use, distribution, and reproduction in any medium, provided the original author and source are credited. 